\title{
A Survey on Analog-to-Digital Converter Performance with Respect to Ionizing Radiation
}

\author{
Steffen Mueller, Robert Weigel, and Alexander Koelpin
}

\begin{abstract}
This paper discusses radiation performance evolution of analog-to-digital converters, based on a comprehensive empirical analysis of both, experimental reports and datasheet specifications including commercial devices, radiation hardened parts, and scientific converters.
\end{abstract}

Index Terms-Analog-to-digital converter, survey, radiation effects, COTS, SEE, TID.

\section{Motivation}

Radiation effects within analog-to-digital converters (ADCs) have been subject to research since more than 25 years [1]. Since ADCs are complex devices that integrate both, analog and digital circuitry, while being available at different performance classes, architectures, and corresponding semiconductor technologies, assessment of radiation response becomes not intuitive. Although numerous results on individual parts have been reported by the radiation effects community, the big picture regarding state-of-the-art radiation performance is still missing. This is especially severe for system designers as ADC is a key component of digital signal processing (DSP) systems that significantly determines system's architecture and overall performance. Furthermore, there is a lack for comparative analysis of single results from previous investigations in order to allow derivation of trends and benchmarks with respect to advances in circuit and semiconductor technology. Goal of this survey is to compile results for a representative number of distinct devices in order to unveil the evolution and the practical limits of ADC radiation performance to the present.

\section{COMPILATION}

\section{A. Data Collection}

In total, 149 distinct devices were identified for which radiation data in terms of single-event effects (SEE) or total ionizing dose (TID) results are available. The compilation comprises different device classes grouping into commercial off-the-shelf (COTS) components, military rated (mil) devices, radiation hardened (radhard) parts, and scientific converter implementations. Simulation-only results were excluded from this study due to their limited practical relevance and lack for comparability. Data was gathered from scientific publications

S. Mueller and R. Weigel are with the Institute for Electronics Engineering (LTE) at the Friedrich-Alexander University of ErlangenNuremberg (FAU), Cauerstr. 9, 91058 Erlangen, Germany. E-mail: \{ste.mueller | robert.weigel\} @ fau.de

A. Koelpin is with the Chair for General Electrical Engineering and Metrology at the Brandenburg University of Technology CottbusSenftenberg (BTU), Siemens-Halske-Ring 14, 03046 Cottbus, Germany. Email: alexander.koelpin@b-tu.de which have been reported by the radiation effects community since early 1980s, and supplemented by datasheet specifications in the case of commercially available radhard parts.

\section{B. Functional Performance Limits}

Fig. 1 shows the total market of 2642 commercial devices that are available in 2016 from leading manufacturers versus the compilation, yielding a coverage of less than $6 \%$. Using (1) as a metric for functional performance (i. e. neglecting any radiation criteria) according to Walden's approach [2],

$$
P=2^{N} \cdot f_{S}
$$

where $N$ refers to the stated resolution and $f_{s}$ to the maximum sampling rate, follows that the compilation is limited by the isometric slope $P=2^{13} \cdot 10^{9}$ while the total market is bounded by $P=2^{16} \cdot 10^{9}$. In other words, the compilation's functional performance lags 3 bit or factor 8 in sampling rate, respectively. This corresponds to a technology gap of 12 years, considering that the speed-resolution product given by (1) typically doubles every four years [3]. The gap is expected to originate from the slow adoption rate for new technology nodes by the radiation community [4], and was similarly found for other devices, such as microprocessors, as well [5].

From Fig. 1 get obvious that vertical resolution is virtually bounded by 12 bit for scientific types, while the majority of radhard parts is limited by 14 bit. The range beyond 16 bit is covered by COTS only. One the other hand, radhard converters are available at GSa/s performances where equivalent COTS have been barely subject to investigations.

\section{Device Classes, Converter Architectures, and Technologies}

COTS and military rated devices contribute by $72 \%$ to this study, owed to the empirical approach for up-screening of candidate devices that are not hardened by design. Investigations on scientific converters have $10 \%$ share only, which might be a consequence of huge efforts for application specific integrated circuit (ASIC) design and testing, together with the fact that results are often under disclosure, thus not available to the general public. Radhard devices have $18 \%$ share only, which underlines their limited coverage of performance range.

Pipeline $(42 \%)$ and successive approximation register (SAR) converters (45\%) taken together cover the majority of devices, since these architectures are popular for mainstream converter implementations. Whereas sigma-delta modulator $(5 \%)$, flash $(4 \%)$, and folding/interpolation converters (3\%) are rather uncommon as they apply either for high resolution or 




Figure 1. Total market of commercial ADC in 2016 (black dots) versus compilation of devices for which radiation data is available (colored symbols).

high-speed applications. Thus knowledge on radiation effects mainly reduces to Pipline and SAR types.

More than two thirds of devices under investigation are fabricated in CMOS technology. Thanks to its good scaling potential together with advances in circuit design such as switched-capacitor technology, CMOS qualifies for a broad range of today's converters. Bipolar $(21 \%)$ and BiCMOS $(11 \%)$ devices typically appear either in legacy or in highspeed converters where bipolar outperforms CMOS regarding required switching capabilities.

According to Fig. 2 the predominant technology node is $180 \mathrm{~nm}$, but even some legacy technologies are reasonably investigated and still in use. Unfortunately, technology insight is rather a blind spot of COTS testing, hence publications often lack for feature size information. Nevertheless, feature sizes could be determined for $70 \%$ of devices of this compilation, allowing to draw reasonable trends on scaling.

\section{RADIATION PERFORMANCE ANALYSIS}

\section{A. Total Ionizing Dose}

1) Scaling: Fig. 3 depicts feature size versus TID threshold for functional failure ${ }^{1}$ with respect to different device classes

\footnotetext{
${ }^{1}$ In this context, functional failure is considered as the threshold dose up to which the device is usable to its intended functionality, including possible parameter degradation. For radhard devices it is regarded as the minimum specified dose for radiation tolerance given in the datasheet.
}

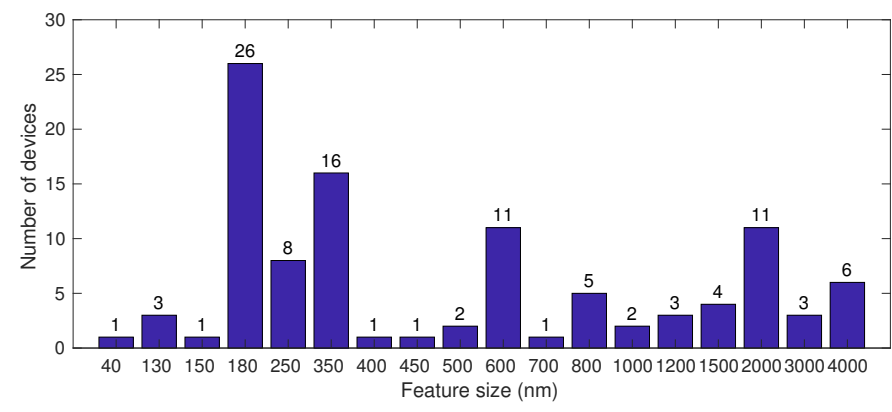

Figure 2. Compilation's distribution by feature size. and process technologies. Apparently TID is inversely proportional to feature size $L$ squared. This trend can be related to the effect of gate threshold voltage shift $\Delta V_{\text {th }}$ due to charge buildup in oxides which is considered as the primary mechanism for degradation in MOS platforms [6]. It is known from [7] that $\Delta V_{\mathrm{th}}$ is proportional to gate oxide thickness $t_{\mathrm{ox}}$ according to

$$
\Delta V_{\mathrm{th}} \propto t_{\mathrm{ox}}^{2}
$$

Regarding $\Delta V_{\text {th }}$ as a measure for radiation-induced damage, it follows that TID tolerance is the reciprocal of $\Delta V_{\mathrm{th}}$. Taking Dennard's concept of constant field scaling [8] into account, i. e. all physical dimensions of transistors (including $L$ and $\left.t_{\text {ox }}\right)$ are reduced by the same factor, Eq. (2) can be rewritten to

$$
\mathrm{TID} \propto \frac{1}{L^{2}}
$$

From Fig. 3 gets obvious that commercial CMOS technologies clearly benefit from scaling, although performances scatter partly by factor 10 from the expected projection. Scientific converters mainly stick close to the trend. Radhard devices are not expected to follow the trend strictly, as these parts are usually specified and optimized for TID tolerances between 1 to $3 \mathrm{kGy}$ which are common values for space qualified devices.

Eq. (3) is expected to be not necessarily valid for deep sub-micron nodes $(<100 \mathrm{~nm})$, as additional effects may arise for these technologies affecting TID response, e.g. tunnel annealing in very thin oxides $(<20 \mathrm{~nm})$ [9] or increased $1 / \mathrm{f}$ noise that scales with $1 / L^{3}$ in MOS devices [10].

2) Device Properties: Fig. 4 shows device properties in terms of sampling rate and resolution compared to TID for functional failure, where closed symbols represent COTS and open symbols denote either radhard or scientific parts. TID tolerance appears to be relatively independent from device resolution, whereas it seems to increase with sampling rate. The latter is consequence of the fact that high-speed devices are usually based on latest technologies with small feature sizes. 


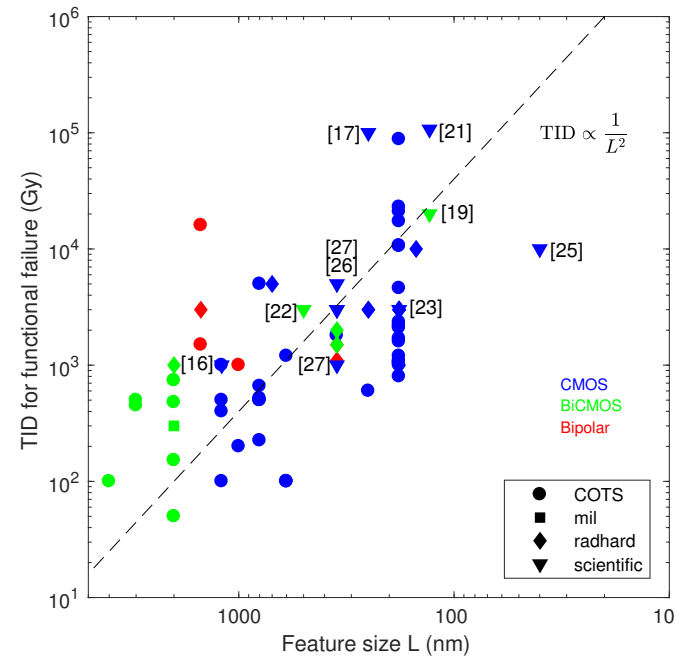

Figure 3. Feature size versus TID threshold for functional failure with respect to different device classes and technologies (dashed line is not a fit).

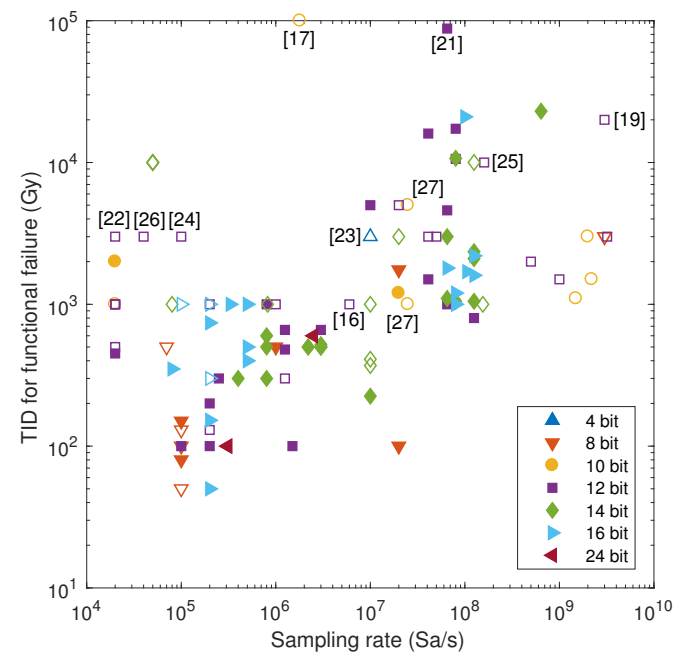

Figure 4. Sampling rate versus TID for functional failure with respect to different resolutions and device classes (filled symbols represent COTS, open symbols denote radhard or scientific parts).

\section{B. Single-Event Effects (SEE)}

Fig. 5 shows the LET threshold for single event latchup (SEL). Although being widespread, the expected trend of increased SEL sensitivity with respect to smaller feature sizes is observable. Best performers of COTS category achieve an LET threshold of $60 \mathrm{MeVcm}^{2} \mathrm{mg}^{-1}$ at small feature sizes.

\section{Combined Performance}

Fig. 6 shows the combined TID and SEL performance from previous sections. The leading edge towards the upper right corner is given through scientific converters and CMOS radhard parts. As expected COTS are widespread and bounded by upper performance limit at LET of $\sim 85 \mathrm{MeVcm}^{2} \mathrm{mg}^{-1}$ and TID of $1 \mathrm{kGy}$. A few bipolar COTS are fairly competitive compared to dedicated radhard parts by achieving LET immunity at $120 \mathrm{MeVcm}^{2} \mathrm{mg}^{-1}$ together with high TID at $10 \mathrm{kGy}$.

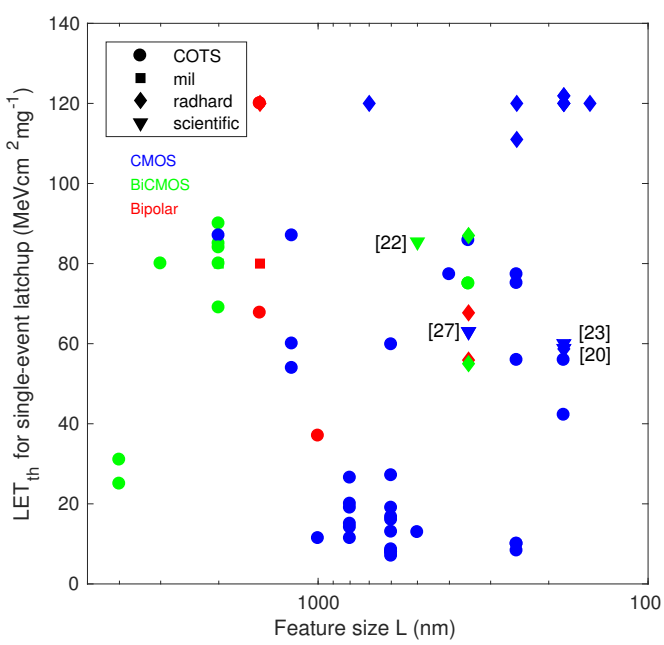

Figure 5. LET threshold for single event latchup versus feature size with respect to different device classes and technologies.

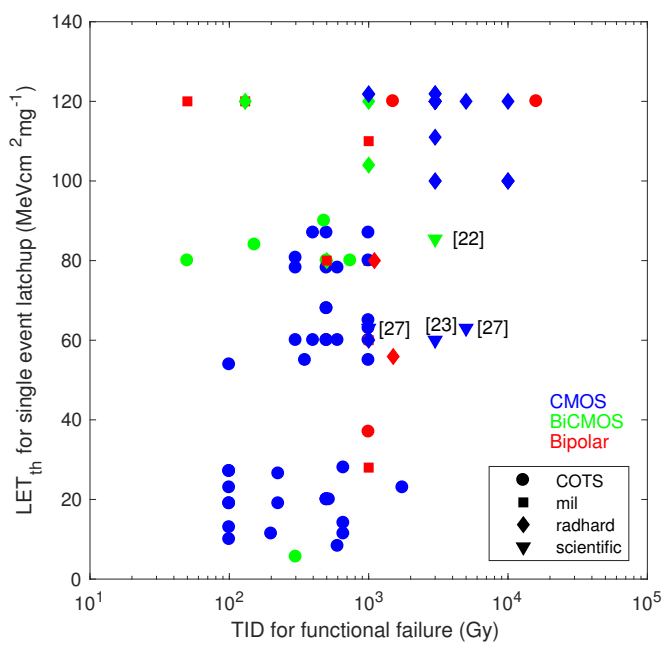

Figure 6. TID threshold for functional failure versus LET threshold for single event latchup with respect to different device classes and technologies.

\section{Comparability}

Correlation of large amount of data from different investigations makes a discussion on comparability necessary.

\section{A. Device Classes}

Comparison of different device classes is not fair per se. COTS are intentionally not hardened by design, while radhard and scientific converters apply sophisticated circuit techniques together with special process technology in order to mitigate radiation effects. In addition, radhard devices feature a specified performance with a design margin usually on top, thus their absolute performance may be underestimated. Scientific converters are typically at early experimental stage where detailed device characterization (electrical, environmental, radiation) is often pending, making comparison difficult.

\section{B. Experimental Conditions}

COTS and scientific devices are usually experimentally investigated by different research groups and at distinct facilities. 
Several factors introduce variability to experiments that may alter radiation responses and lead to over- or underestimation of results, e.g. facility configuration (calibration, dosimetry, ambient temperature), beam factors (uniformity, particle species and energy spectra, dose rate, flux, angle of incidence), electrical configuration (bias vs. unbiased operation, static vs. dynamic input), die preparation (delidding, burn-in), test procedure (standardized vs. non-standardized), data acquisition method (manually vs. online), definition of failure criteria and thresholds.

\section{CONCLUSION}

Assessment of ADC radiation performance is complex due highly individualized investigations on a broad range of devices. Based on a large compilation of devices, trends and limits could be derived that make ADC performance evolution and state-of-the-art visible. However, discussion on comparability is necessary when correlating results. As far as the authors are aware, this is the most comprehensive collection and comparative analysis on ADC radiation performance to this date that may serve as baseline for future investigations.

\section{REFERENCES}

[1] T. L. Turflinger and M. V. Davey, "Understanding single event phenomena in complex analog and digital integrated circuits," IEEE Transactions on Nuclear Science, vol. 37, no. 6, pp. 1832-1838, 1990.

[2] R. H. Walden, "Analog-to-digital converter survey and analysis," IEEE Journal on Selected Areas in Communications, vol. 17, no. 4, pp. 539$550,1999$.

[3] B. Murmann, "A/D converter trends: Power dissipation, scaling and digitally assisted architectures," IEEE Custom Integrated Circuits Conference, pp. 105-112, 2008.

[4] P. E. Dodd, M. R. Shaneyfelt, J. R. Schwank, and J. A. Felix, "Current and future challenges in radiation effects on CMOS electronics," IEEE Transactions on Nuclear Science, vol. 57, no. 4, pp. 1747-1763, 2010.

[5] R. C. Lacoe, J. V. Osborn, R. Koga, S. Brown, and D. C. Mayer, "Application of hardness-by-design methodology to radiation-tolerant ASIC technologies," IEEE Transactions on Nuclear Science, vol. 47, no. 6, pp. 2334-2341, 2000.

[6] T. L. Turflinger, M. V. Davey, and J. P. Bings, "Radiation effects in analog CMOS analog-to-digital converters," IEEE Radiation Effects Data Workshop, pp. 6-12, 1996.

[7] J. M. McGarrity, "Considerations for hardening MOS devices and circuits for low radiation doses," IEEE Transactions on Nuclear Science, vol. 27, no. 6, pp. 1739-1744, 1980.

[8] R. H. Dennard, F. H. Gaensslen, V. L. Rideout, E. Bassous, and A. R. Leblanc, "Design of ion-implanted MOSFET's with very small physical dimensions," IEEE Journal of Solid State Circuits, vol. 9, no. 5, pp. 256-268, 1974.

[9] N. S. Saks, M. G. Ancona, and J. A. Modolo, "Radiation effects in MOS capacitors with very thin oxides at $80^{\circ} \mathrm{K}$," IEEE Transactions on Nuclear Science, vol. 31, no. 6, pp. 1249-1255, 1984.
[10] M.-H. Tsai and T.-P. Ma, "The impact of device scaling on the current fluctuations in MOSFET's," IEEE Transactions on Nuclear Science, vol. 41, no. 11, pp. 2061-2068, 1994.

[11] F. G. Broell and W. J. Barnard, "A radiation-hardened CMOS 8-bit analog-to-digital converter," IEEE Transactions on Nuclear Science, vol. 30, no. 6, pp. 4246-4250, 1983.

[12] F. Baille, G. Borel, B. Commère, R. Roy, C. Delmas, and C. Terrier, "A multi MRad hardened 8 bit / $20 \mathrm{MHz}$ flash ADC," IEEE Transactions on Nuclear Science, vol. 39, no. 3, pp. 401-404, 1992.

[13] S. C. Nystrom, T. R. Smith, W. R. Peterson, D. L. LeFevre, D. T. Butcher, L. Gipson, and M. M. Spanish, "Ultra-low-power, radiationhardened 12-bit analog-to-digital converter for space-based electrooptical sensors," 17th Digital Avionics Systems Conference, vol. 2, pp. H31/1-H31/7, 1998.

[14] A. Rivetti, G. Anelli, F. Anghinolfi, G. Mazza, and F. Rotondo, "A low-power 10-bit ADC in a 0.25 um CMOS: design considerations and test results," IEEE Transactions on Nuclear Science, vol. 48, no. 4, pp. $1225-1228,2001$.

[15] G. Kottaras, N. P. Paschalidis, E. T. Sarris, N. Stamatopoulos, K. Karadamoglou, and V. Paschalidis, "A 10-Bit, low power, successive approximation, digitally auto-zeroed CMOS ADC core for the NASA TRIO smart sensor system on a chip," Analog Integrated Circuits and Signal Processing, vol. 42, no. 2, pp. 113-128, 2005.

[16] E. O. Mikkola and J. D. Cressler, "12-bit, 3 GS/s, radiation-hard timeinterleaved ADC for particle accelerator applications," IEEE Nuclear Science Symposium and Medical Imaging Conference, pp. 687-692, 2012.

[17] R. Koga, C. Paul, D. Romeo, V. Petrosyan, and J. George, "Single event effects sensitivity of 180 and $350 \mathrm{~nm}$ SiGe HBT microcircuits," IEEE Radiation Effects Data Workshop, pp. 1-7, 2013.

[18] J. Kuppambatti, J. Ban, T. Andeen, P. Kinget, and G. Brooijmans, "A radiation-hard dual channel 4-bit pipeline for a 12-bit $40 \mathrm{MS} / \mathrm{s}$ ADC prototype with extended dynamic range for the ATLAS liquid argon calorimeter readout electronics upgrade at the CERN LHC," Journal of Instrumentation, vol. 8, no. 9, p. P09008, 2013.

[19] P. C. Adell, J. Yager, Z. Pannell, J. Shelton, M. M. Mojarradi, B. Blalock, G. Allen, and R. Some, "Radiation hardening of an SiGe BiCMOS wilkinson $\mathrm{ADC}$ for distributed motor controller application," IEEE Transactions on Nuclear Science, vol. 61, no. 3, pp. 1236-1242, 2014

[20] U. Gatti and C. Calligaro, "A family of rad-hard ADC with flash architecture," European Conference on Radiation and its Effects on Components and Systems, pp. 1-5, 2015.

[21] T. H. Kim and H. C. Lee, "Tid effect on a 12-bit 100ksps sar adc designed with a dummy gate-assisted n-mosfet," IEEE Nuclear Science Symposium and Medical Imaging Conference (NSS/MIC), pp. 1-4, 2015.

[22] H. Xu, Y. Zhou, Y. Chiu, D. Gong, T. Liu, and J. Ye, "High-speed, high-resolution, radiation-tolerant SAR ADCs for particle physics experiments," Journal of Instrumentation, vol. 10, no. 4, p. C04035, 2015.

[23] T. H. Kim and H. C. Lee, "Total ionizing dose effects on a 12-bit $40 \mathrm{kS} / \mathrm{s}$ SAR ADC designed with a dummy gate-assisted n-MOSFET," IEEE Transactions on Nuclear Science, vol. 64, no. 1, pp. 648-653, 2017.

[24] F. Qi, T. Liu, H. Liu, C. Zeng, B. Li, F. Zhao, J. Gao, G. Zhang, J. Luo, Z. Han, and Z. Liu, "Comparison study of bulk and SOI CMOS technologies based rad-hard ADCs in space," ESA International Workshop on Analogue and Mixed-Signal Integrated Circuits for Space Applications, 2016

[25] F. Bouyjou, O. Gevin, O. Limousin, and E. Delagnes, "A 32-channel 13b ADC for space applications," IEEE Transactions on Nuclear Science, vol. 64, no. 4, pp. 1071-1079, 2017. 